\title{
Low-Molecular Weight Heparin/Protamine Micro-Nanoparticles Augmented Viability of Human Adipose-Derived Regenerative Cells
}

\author{
Masashi Sakuma $^{1,3}$, Satoko Kishimoto*2,3, Ken-ichi Inoue ${ }^{2,3}$, Ryoichi Sohma ${ }^{2}$, Shigeru Toyoda ${ }^{1,3}$, Hideki Iwaguro ${ }^{1,3}$ \\ and Teruo Inoue ${ }^{* 1,2,3}$
}

${ }^{1}$ Department of Cardiovascular Medicine, Japan

${ }^{2}$ Research Support Center, Japan

${ }^{3}$ Center for Regenerative Medicine, Japan

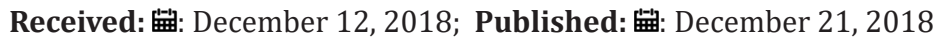

*Corresponding author: Teruo Inoue, Department of Cardiovascular Medicine, Japan and Satoko Kishimoto, Research Support Center, Center for Regenerative Medicine Tochigi, Japanb

\section{Abstract}

Background: Low molecular weight heparin/protamine micro/nanoparticles (LH/P-MPs) has been shown as cell carriers for adipose-derived regenerative cells (ADRCs) to augment cell viability, leading to accelerating angiogenesis in mouse limb ischemia models, compared to ADRCs alone.

Material \& Methods: Human ADRCs were isolated from 3 patients with critical limb ischemia and incubated with and without LH/P-MPs Incubation of ADRCs with LH/P-MPs produced cell aggregation (ADRC/LH/P-MPs aggregates). Serial changes in survived ADRCs were compared between ADRC/LH/P-MPs aggregates and ADRCs alone.

Results: Patients' derived ADRCs promptly decreased cell number in suspension culture, eventually they were eliminated through cell death after seven days. ADRC/LH/P-MPs aggregates remarkably slowed down the cell death.

Conclusion: The LH/P-MPs could enhance the viability of human ADRCs. The LH/P-MPs could be a promising option in ADRCs-based therapeutic angiogenesis in patients with critical limb ischemia.

Keywords: Limb Ischemia; Adipose-Derived Regenerative Cells; Low Molecular Weight Heparin/Protamine Micro/Nanoparticles; Angiogenesis Therapy; Human

\section{Introduction}

Recently stem cell therapies have been widely applied for various diseases refractory against conventional medical as well as surgical treatments. Critical limb ischemia due to peripheral artery disease or Bürger disease is a rational indication for the stem cell implantation to induce neovascularization. Bone marrow-derived stem cells (BMSCs) can improve limb ischemia after injection in experimental limb ischemia models [1] and patients with critical limb ischemia [2]. Mesenchymal stem cells (MSCs) are also known to induce neovascularization in a hindlimb ischemia model [3]. Adipose tissue is abundant in the human body and is constantly reorganized through angiogenesis. Therefore, this tissue is an ideal source of angiogenic MSCs. It has been shown that adipose-derived regenerative cells (ADRCs), which contain not only MSCs but also various regenerative cells such as endothelial progenitor cells, have characteristics similar to those of BMSCs [4]. The implantation of in vitro cultured ADRCs in the mouse hindlimb ischemia model was effective for its proangiogenic action [5]. In addition, the safety and effectiveness of autologous ADRCs implantation in patients with critical limb ischemia has been reported [6].

Recently an automated cell-processing system for the isolation of ADRCs has been developed and its safety and reproducibility have been assessed [7]. We have recently reported low molecular weight heparin/protamine micro/nanoparticles (LH/P-MPs) can be used as cell carriers for autologous ADRCs in mouse hindlimb ischemia model. The LH/P-MPs bind to the cell surface of ADRCs through heparin-binding proteins such as integrins. The interaction 
of the ADRCs with LH/P-MPs results in the formation of ADRC/ LH/P-MP aggregates, sustaining survival of the cells. In the inbred mouse hindlimb ischemia model, these aggregates efficiently increase cell viability in vitro and injection of them augmented neovascularization in vivo, compared to ADRCs alone. As a result, the ADRC/LH/P-MP aggregates were more effective in preventing the loss of ischemic hindlimbs compared with ADRCs alone [8]. However, the superior effect of ADRC/LH/P-MP aggregates on ameliorating critical limb ischemia over ADRCs alone has not been confirmed in human. In the present study, we investigated whether the LH/P-MPs augment cell viability in human ADRCs in vitro.

\section{Material and Methods}

ADRCs were isolated from periumbilical subcutaneous adipose tissues collected by a liposuction procedure in 3 patients with critical limb ischemia (Case 1: 51yo male; progressive systemic sclerosis, Case 2: 70 yo female; progressive systemic sclerosis and Case 3: 45yo male; Bürger disease), who were planned to undergo the stem cell therapy for ischemic limbs. For the isolation of ADRCs, we used an automated cell-processing system, Cytori Celution device (Cytori Therapeutics Inc., San Diego, California). The stem cell therapy was performed under the approval of local committee for regenerative medicine and an informed consent regarding the present study was obtained from each patient. The LH/P-MPs were synthesized as described previously [9]. Briefly, $0.3 \mathrm{~mL}$ of protamine sulfate solution (10mg/mL; Mochida Pharmaceutical Co., Tokyo, Japan) was added dropwise to $0.7 \mathrm{~mL}$ of a low-molecular-weight heparin solution, Dalteparin sodium (6.4 mg/mL; Kissei Pharmaceutical Co., Tokyo, Japan) and vortexed for approximately $2 \mathrm{~min}$. The mixed LH/P-MPs were then washed twice with phosphate-buffered saline to remove nonreactants using centrifugation at $4,900 \mathrm{~g}$ for $5 \mathrm{~min}$, and the precipitates were finally resuspended in $1 \mathrm{~mL}$ of Dulbecco's modified Eagle's medium (DMEM; Life Technologies Oriental, Tokyo, Japan).
Subsequently, more than 6mg of dry LH/P-MPs were obtained from $1 \mathrm{~mL}$ of the resuspended LH/P-MPs solution. In the final preparation step, 60mg of LH/P-MPs were resuspended in $1 \mathrm{~mL}$ of DMEM. ADRCs isolated from each patient were incubated in DMEM containing heat-inactivated fetal bovine serum with and without LH/P-MPs (about $1.4 \mathrm{mg}$ of dried particles/mL) in $15 \mathrm{~mL}$ polypropylene conical tubes with occasional shaking at $37^{\circ} \mathrm{C}$ for the indicated time periods. Incubation of ADRCs with LH/P-MPs produced cell aggregation (ADRC/LH/P-MPs aggregates). Survived ADRCs were counted at the next day, and 2 days, 3 days and 7 days after the first day of incubation for $5 \times 103$ cells by the 12 experiments in each sample of the 3 patients. Statistical analyses were carried out using SPSS software (IBM, Armonk, NY, USA). To detect the main effect by LH/P-MPs, we adopted Fisher's least significant difference procedure (analysis of variance and student's t test as a post hoc). $\mathrm{P}$ value less than 0.01 were considered significant.

\section{Results}

Patients' derived ADRCs promptly decreased cell number in suspension culture, eventually they were eliminated through cell death after seven days. Addition of LH/P-MPs produced aggregation with ADRCs, remarkably slowed down the cell death. Such a protection effect against cell death was reproduced in three independent patients. The number of survived ADRC was significantly higher in ADRC/LH/P-MPs aggregates compared to ADRCs alone at the next day (Case 1: $4.53 \pm 0.34$ vs $1.49 \pm 0.29$ $\times 103, \mathrm{P}<0.01$; Case 2 : $4.10 \pm 0.44$ vs $1.28 \pm 0.39 \times 103, \mathrm{P}<0.01$; and Case 3: $4.33 \pm 0.35$ vs $1.83 \pm 0.56 \times 103, \mathrm{P}<0.01$ ), at the 2 days (Case 1: $4.11 \pm 0.38$ vs $1.00 \pm 0.28 \times 103, \mathrm{P}<0.01$; Case 2 : $3.38 \pm 0.37$ vs $0.75 \pm 0.26 \times 103, \mathrm{P}<0.01$; and Case 3 : $3.75 \pm 0.41$ vs $1.04 \pm 0.48 \times 103$, $\mathrm{P}<0.01$ ) and at the 3 days (Case 1: $3.13 \pm 0.37$ vs $0.62 \pm 0.26 \times 103$, $\mathrm{P}<0.01$; Case 2: $2.45 \pm 0.30$ vs $0.40 \pm 0.19 \times 103, \mathrm{P}<0.01$; and Case 3 : $2.72 \pm 0.50$ vs $0.51 \pm 0.26 \times 103, \mathrm{P}<0.01$ ) (Figure 1 ).

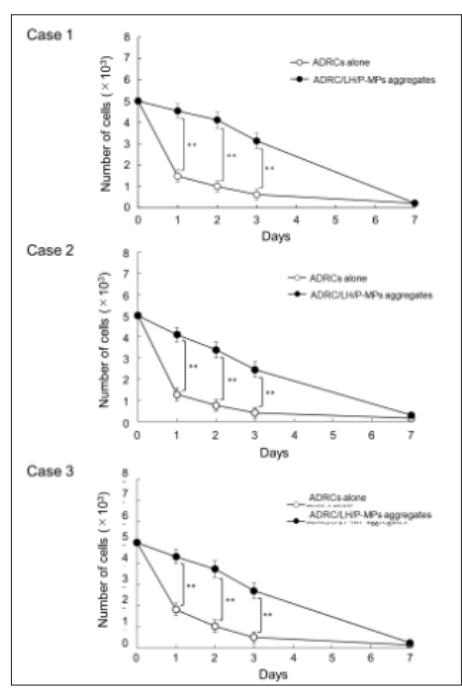

Figure 1: Serial changes in viability of ADRCs. Patients' derived ADRCs promptly decreased cell number in suspension culture, eventually they were eliminated through cell death after seven days. Addition of LH/P-MPs produced aggregation with ADRCs, remarkably slowed down the cell death. Such a protection effect against cell death was reproduced in three independent patients. ADRC, adipose-derived regenerative cell; LH/P-MPs, low molecular weight heparin/protamine micro/nanoparticles **P<0.01. 


\section{Discussion}

In the present study, we observed that cell viability of ADRCs isolated from human subcutaneous adipose tissues was augmented, when the LH/P-MPs were added to the ADRCs and ADRC/LH/PMPs aggregates were formed, compared with the ADRCs alone in all of 3 patients with critical limb ischemia. Survived cell number was reduced to approximately $30 \%$ at the next day, $20 \%$ at the 2 days and only $10 \%$ at the 3 days after incubation in case of ADRCs alone, indicating that ADRCs hardly sustain cell number due to lack of extracellular scaffold. On the other hand, addition of LH-P-MPs rescued such a rapid cell death and the ratio increased to $90 \%$ (next day), $80 \%$ (2 days) and $70 \%$ (3 days), respectively. The ADRCs have the potential to differentiate into various cell lineages, including vascular cells [10]. In addition, cultured ADRCs secrete various angiogenic growth factors, such as basic fibroblast growth factor, hepatocyte growth factor, and vascular endothelial growth factor as well as cytokines [11-13]. Angiogenetic mechanism of stem cell therapy such as ADRCs therapy is mainly based on paracrine effects by these angiogenic factors [14].

Apart from the protection effects as a cellular scaffold, LH/PMPs can immobilize and stabilize various angiogenic factors through heparin binding motifs. As a results, the beneficial effects of LH/P-MPs are two-fold: Sustaining survival of ADRCs per se and stabilization of various angiogenic factors or cytokines. Therefore, the precise mechanisms of augmenting angiogenesis are suggested to be as follows. First, injected ADRCs are protected from cell death by LH/P-MPs and continuously release of multiple angiogenic factors. Next, these angiogenic factors are adsorbed onto LH/PMPs, permitting their stabilization and bioavailability. Such dual effects are considered to be critical for augmented angiogenesis in vivo [8]. In the present study, we demonstrated that the LH/P-MPs could enhance the survival of patients' derived ADRCs. Both lowmolecular-weight heparin and protamine are agents available for clinical use and the safety concern using LH/P-MPs is relatively low. Therefore, LH/P-MPs could be a promising option in therapeutic angiogenesis as cellular scaffolds for ADRCs in human.

\section{References}

1. Asahara T, Murohara T, Sullivan A, Silver M, van der Zee R, et al. (1997) Isolation of putative progenitor endothelial cells for angiogenesis. Science 275(5302): 964-967.

\section{ISSN: 2574-1241}

DOI: $10.26717 / B J S T R .2018 .12 .002265$

Teruo Inoue. Biomed J Sci \& Tech Res

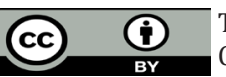

This work is licensed under Creative Commons Attribution 4.0 License

Submission Link: https://biomedres.us/submit-manuscript.php
2. Tateishi Yuyama E, Matsubara H, Murohara T, Ikeda U, Shintani S, et al. (2002) Therapeutic angiogenesis for patients with limb ischaemia by autologous transplantation of bone-marrow cells: a pilot study and a randomised controlled trial. Lancet 360(9331): 427-435.

3. Al Khaldi AH, Al Sabti J, Galipeau J, Lachapelle K (2003) Therapeutic angiogenesis using autologous bonemarrow stromal cells: Improved blood flow in a chronic limb ischemia model. Ann Thorac Surg 75(1): 204-209.

4. Lee RH, Kim B, Choi I, Kim H, Choi HS, et al. (2004) Characterization and expression analysis of mesenchymal stem cells from human bone marrow and adipose tissue. Cell Physiol Biochem 14(4-6): 311-324.

5. Moon MH, Kim SY, Kim YJ, Kim SJ, Lee JB, et al. (2006) Human adipose tissue-derived mesenchymal stem cells improve postnatal neovascularization in a mouse model of hindlimb ischemia. Cell Physiol Biochem 17(5-6): 279-290.

6. Lee HC, An SG, Lee HW, Park JS, Cha KS, et al. (2012) Safety and effect of adipose tissue-derived stem cell implantation in patients with critical limb ischemia: a pilot study. Circ J 76(7): 1750-1760.

7. Inoue K, Nomura H, Sohma R, Akimoto K, Kobayashi N, et al. (2014) Feasibility of exploiting CelutionTM system in autologous cell therapy in Dokkyo Medical University Hospital: safety and reproducibility. Dokkyo J Med Sci 41(1): 7-12.

8. Kishimoto S, Inoue $\mathrm{K}$, Nakamura S, Hattori H, Ishihara M, et al. (2016) Low-molecular weight heparin protamine complex augmented the potential of adipose-derived stromal cells to ameliorate limb ischemia. Atherosclerosis 249: 132-139.

9. Mori Y, Nakamura S, Kishimoto S, Kawakami M, Suzuki S, et al. (2010) Preparation and characterization of low-molecular-weight heparin/ protamine nanoparticles (LMW-H/P NPs) as FGF-2 carrier. Int J Nanomed 5: 147-155.

10. Gimble JM, Katz AJ, Bunnel BA (2007) Adipose-derived stem cells for regenerative medicine. Circ Res 100(9): 1249-1260.

11. Nakagami H, Morishita R, Maeda K, Kikuchi Y, Ogihara T, et al. (2006) Adipose tissue-derived stromal cells as a novel option for regenerative cell therapy. J Atheroscler Thromb 13(2): 77-81.

12. Nambu M, Ishihara M, Kishimoto S, Yanagibayashi S, Yamamoto N, et al. (2011) Stimulatory effect of autologous adipose tissue-derived stromal cells in an atelocollagen matrix on wound healing in diabetic $\mathrm{db} / \mathrm{db}$ mice. J Tissue Eng 2011: 158105.

13. Takikawa $M$, Nakamura $S i$, Nakamura $S$, Nambu $M$, Ishihara $M$, et al. (2011) Enhancement of vascularization and granulation tissue formation by growth factors in human platelet-rich plasma-containing fragmin/protamine microparticles. J Biomed Mater Res B Appl Biomater 97(2): 373-380.

14. Hsiao ST, Lokmic Z, Peshavariya H, Abberton KM, Dusting GJ, et al. (2013) Hypoxic conditioning enhances the angiogenic paracrine activity of human adipose-derived stem cells. Stem Cells Dev 22(10): 1614-1623.

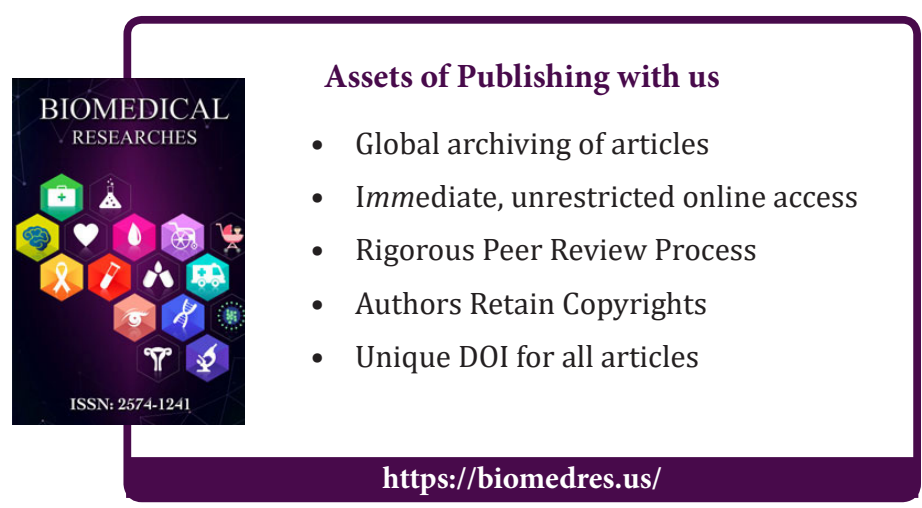

Cite this article: Masashi S, Satoko K, Ken-ichi I, Ryoichi S, Shigeru T, Hideki I, Teruo I. Low-Molecular Weight Heparin/Protamine Micro-Nanoparticles Augmented Viability of Human Adipose-Derived Regenerative Cells. Biomed J Sci \& Tech Res 12(3)-2018. BJSTR. MS.ID.002265. DOI: 10.26717/ BJSTR.2018.12.002265. 